DOE/ER/40427-15-N94, TMU-NT940801

\title{
Photon Vector-Meson Coupling and Vector Meson Properties at Low Temperature Pion Gas
}

\author{
Su Houng Lee, 2 , Chungsik Song ${ }^{3}$ and Hiroyuki Yabu四 \\ ${ }^{1}$ Department of Physcis, Yonsei University, Seoul 120-749, Korea \\ ${ }^{2}$ Department of Physics, FM-15, University of Washington, Seattle, WA 98195, USA \\ ${ }^{3}$ Cyclotron Institute, Texas A\&MM University, College Station, TX 77843, USA \\ ${ }^{4}$ Department of Physics, Tokyo Metropolitan University, Hachioji, Tokyo 192, Japan
}

(August 10 1994)

\begin{abstract}
The vector and axial vector current mixing phenomena at low temperature pion gas by Dey, Eletsky and Ioffe, leads to the low temperature correction of the photon-vector meson coupling $\left(g_{\rho}\right)$ at order $\epsilon=T^{2} / 6 f_{\pi}^{2}$ and the $\rho$ meson mass at order $\epsilon^{2}$. We show how this low temperature theorems involving the photon and vector mesons are satisfied in the chiral models based on hidden gauge symmetry and the massive Yang-Mills approach with an explicit $a_{1}$ meson. We discuss possible phenomenological consequences of the low temperature corrections in RHIC experiments.
\end{abstract}


For many years, dilepton spectrum from Relativistic Heavy Ion Collision (RHIC) has attracted a lot of interest [1]. This is so because once produced, they will predominantly escape from the collision region without further interaction carrying information about the hot initial stages of the collision. Of particular interests are the vector meson resonance regions because model calculations show definite relations between chiral symmetry restoration at finite temperature or density to vector meson masses. If this is so, chiral symmetry restoration could be directly observable through shift of vector meson peak positions in the dilepton spectrum.

The vector meson properties and dilepton spectrum at finite temperature have been studied in various ways [2 8]. Recently, it was realized by Dey et.al. [DEI] [3] that in the lowest order in $\epsilon=T^{2} / 6 f_{\pi}^{2}$, where $f_{\pi}=93 \mathrm{MeV}$, there is no change in the vector meson masses and only mixing between vector and axial vector correlator takes place. They obtained the result based only on the PCAC and current algebra. The low temperature limit of the QCD sum rule calculations at finite temperature indeed satisfies this constraint [7.99.

In the effective model approach, chiral perturbation theory [10,[1], although successful in reproducing systematically the low energy hadron physics, is not able to reproduce the higher energy region including the resonances. Therefore, effective chiral models with explicit $\rho$ and $a_{1}$ meson degrees of freedom were used to calculate the vector meson properties and dilepton spectrum from the low temperature pion gas 四. This way, although we can not generate the $\rho$ meson pole, the changes of the vector meson properties due to low temperature pionic effect could be obtained in a chirally invariant way. In this respect, at low temperature, the effective model approaches should also be consistent with the mixing phenomena while the $\rho$ meson mass changes at the order $\epsilon^{2}$.

The purpose of this letter is the following. First, we will summarize the mixing phenomena by DEI and its implications to the effective photon vector-meson coupling and the vector meson mass. Second, we will show how the correct temperature dependence of the photon vector meson coupling and the vector meson mass come out in the effective chiral 
lagrangians. Finally, we will comment on possible physical consequences in RHIC.

Let us consider the thermal average of an operator $\mathcal{O}$ :

$$
\langle\mathcal{O}\rangle_{T}=\sum_{n}\langle n|\mathcal{O} \exp (-H / T)| n\rangle
$$

Here the sum is over the full set of eigenstates of the hamiltonian $H$. At low temperature $\left(T<m_{\pi}\right)$, we shall take into account only the vacuum state and the lowest excitation mode of the hadron gas, the pions. Then the thermal average can be approximated by

$$
\langle\mathcal{O}\rangle_{T}=\langle\mathcal{O}\rangle_{T=0}+\sum_{a=1}^{3} \int \frac{d^{3} p}{2 \omega(2 \pi)^{3}}\left\langle\pi^{a}(p)|\mathcal{O}| \pi^{a}(p)\right\rangle n_{B}(\omega / T),
$$

where $\omega=\sqrt{p^{2}+m_{\pi}^{2}}$, $a$ denotes the isospin index and $n_{B}(x)=\left[e^{x}-1\right]^{-1}$ is the BoseEinstein distribution. We have used the covariant normalization for the state vector here $\left\langle\pi^{a}(p) \mid \pi^{b}\left(p^{\prime}\right)\right\rangle=2 \omega(2 \pi)^{3} \delta^{a b} \delta^{3}\left(p-p^{\prime}\right)$.

Eq.(2) contains the first non-trivial term of the expansion by the pion number density at $T \neq 0$ and is a good approximation up to $T \sim 150 \mathrm{MeV}$ 12, 13. For larger $T$, the effect of higher powers of the pion density and other massive excitations $(K, \eta, \ldots)$ start to become important. The matrix element can be evaluated in the soft pion limit such that

$$
\left\langle\pi^{a}(p)|\mathcal{O}| \pi^{a}(p)\right\rangle=-\frac{1}{f_{\pi}^{2}}\left\langle 0\left|\left[\mathcal{F}_{5}^{a}\left[\mathcal{F}_{5}^{a}, \mathcal{O}\right]\right]\right| 0\right\rangle+O\left(m_{\pi}^{2}\right)
$$

where $\mathcal{F}_{5}^{a}$ is the axial charge operator. Eq.(2) with eq.(3) when applied to chiral order parameter, gives the leading temperature correction $\langle\bar{q} q\rangle_{T}=\langle\bar{q} q\rangle\left(1-\frac{3}{4} \epsilon\right)$, with $\epsilon=T^{2} / 6 f_{\pi}^{2}$ for $\mathrm{SU}(2)$ and as given by the low temperature theorem of chiral perturbation in ref [12].

Let us now look at the time ordered vector correlator at finite temperature

$$
\Pi_{\mu \nu}^{V}(q, T)=i \int d^{4} x e^{i q x}\left\langle T\left[V_{\mu}^{a}(x) V_{\nu}^{a}(0)\right]\right\rangle_{T}
$$

The vector current is $V_{\mu}^{a}=\bar{q} \gamma_{\mu} \frac{\tau^{a}}{2} q$ with $\tau^{a}$ being the $\mathrm{SU}(2)$ isospin matrix normalized as $\operatorname{Tr}\left[\tau^{a} \tau^{b}\right]=2 \delta^{a b}$. DEI [3] showed that in the leading order in $\epsilon, \Pi_{\mu \nu}^{V}$ mixes with the corresponding axial correlator $\Pi_{\mu \nu}^{A}$, such that

$$
\begin{aligned}
& \Pi_{\mu \nu}^{V}(q, T)=(1-\epsilon) \Pi_{\mu \nu}^{V}(q, 0)+\epsilon \Pi_{\mu \nu}^{A}(q, 0) \\
& \Pi_{\mu \nu}^{A}(q, T)=(1-\epsilon) \Pi_{\mu \nu}^{A}(q, 0)+\epsilon \Pi_{\mu \nu}^{V}(q, 0) .
\end{aligned}
$$


This mixing appears only in isospin triplet channel. For the correlator of isospin singlet vector current $V_{\mu}^{\omega}=\frac{1}{2}\left(\bar{u} \gamma_{\mu} u+\bar{d} \gamma_{\mu} d\right)$, however, there is no effect to this order;

$$
\Pi_{\mu \nu}^{S V}(q, T)=\Pi_{\mu \nu}^{S V}(q, 0) .
$$

This is so because two pions can not couple to $\omega$ current.

Let us now look at the imaginary part of the vector correlators near the $\rho, \omega$ meson resonance region. Due to the mixing effect, the strength of the $\rho$ peak is reduced while the pole position is not changed. The reason for the later is due to the vanishing of the $\rho-\pi$ scattering length in the soft pion limit (Adler's zero). The change of the residue of the $\rho$ meson pole can be effectively understood by the change of the coupling constant $\left(g_{\rho}\right)$ of the vector current to $\rho$ meson at finite temperature. In the resonance region, as can be seen in Fig. 1. a, the external current coupling to the $\rho$ meson is reduced by thermal pions

$$
g_{\rho}^{2}(T)=(1-\epsilon) g_{\rho}^{2}+O\left(\epsilon^{2}\right) .
$$

Looking at the imaginary part near the $a_{1}$ meson resonance, we also note that the vector current will couple to the axial mesons to lowest order in $\epsilon$ (Fig. 1. b). This interpretation is also consistent with the origin of the mixing. The interaction of two pions with one current decreases the contributions of the initial channel. Admixture of opposite parity channel arises when the two pions interact with two currents at points 0 and $x$.

It should be emphasized that the mixing effect is a consequence of chiral symmetry and current algebra so that it has to be satisfied in the low temperature limit of any model calculation; i.e. when only the lowest thermal pion contributions are taken into account in the thermal average.

In the literature, there are two known methods of introducing the vector mesons and the external photon field into the chiral lagrangian. These are the massive Yang-Mills approach (MYMA) and the hidden gauge approach (HGA). In the MYMA, the $\rho$ and the $a_{1}$ are introduced as external gauge fields of the chiral group and the photon field is introduced via VMD [14]. In the HGA, the vector meson is introduced as the gauge field of the hidden local 
symmetry and the photon is introduced as the external gauge field [15]. These two methods have been shown to be gauge equivalent [16,17] and give identical results [18] if both the $\rho$ and $a_{1}$ are included.

Starting from an effective lagrangian, the lowest order temperature corrections are obtained by considering the thermal pion loops. In this respect, it is similar to calculating the loop correction in chiral perturbation theory, but since we are interested in the temperature correction only, no infinities occur and no additional higher derivative terms are needed. The additional momentum factor comes in with the temperature and gives correction to the tree level parameters.

Let us consider the chiral lagrangian of Bando et.al. [15], which is based on the $\left[\mathrm{SU}(2)_{\mathrm{L}} \times \mathrm{SU}(2)_{\mathrm{R}}\right]_{\text {global }} \times\left[\mathrm{SU}(2)_{V}\right]_{\text {local }}$ "linear" sigma model. It is constructed with two $\mathrm{SU}(2)$-matrix valued variables $\xi_{L}(x)$ and $\xi_{R}(x)$, which transforms as $\xi_{L, R}(x) \rightarrow \xi_{L, R}^{\prime}(x)=$ $h(x) \xi_{L, R} g_{L, R}^{\dagger}$ under $h(x) \in\left[\mathrm{SU}(2)_{V}\right]_{\text {linear }}$ and $g_{L, R} \in\left[\mathrm{SU}(2)_{L, R}\right]_{\text {global }}$. The vector meson $V_{\mu}$ is introduced as the gauge field of the local symmetry and the photon $\mathcal{B}_{\mu}$ as the external gauge field of the global symmetry. Then the general invariant lagrangian looks as follows,

$$
\begin{aligned}
\mathcal{L} & =\mathcal{L}_{A}+a \mathcal{L}_{V}+\mathcal{L}_{\text {kin }}\left(V_{\mu}, \mathcal{B}_{\mu}\right), \\
\mathcal{L}_{V} & =f_{\pi}^{2} \operatorname{tr}\left[V_{\mu}-\frac{1}{2 i}\left(\mathcal{D}_{\mu} \xi_{L} \cdot \xi_{L}^{\dagger}+\mathcal{D}_{\mu} \xi_{R} \cdot \xi_{R}^{\dagger}\right)\right]^{2}, \\
\mathcal{L}_{A} & =f_{\pi}^{2} \operatorname{tr}\left[\frac{1}{2 i}\left(\mathcal{D}_{\mu} \xi_{L} \cdot \xi_{L}^{\dagger}-\mathcal{D}_{\mu} \xi_{R} \cdot \xi_{R}^{\dagger}\right)\right]^{2} .
\end{aligned}
$$

Here $\mathcal{L}_{A}$ is precisely the gauged CCWZ lagrangian [19] and

$$
\mathcal{D}_{\mu} \xi_{L, R}=\partial_{\mu} \xi_{L, R}+i e \xi_{L, R} \mathcal{B}_{\mu} \tau_{3} / 2
$$

In the "unitary" gauge,

$$
\xi_{L}^{\dagger}(x)=\xi_{R}(x)=e^{i \pi(x) / f_{\pi}} \equiv \xi(x)
$$

and rescaling $V_{\mu} \rightarrow g V_{\mu}$, the effective lagrangian takes the form,

$$
\mathcal{L}=-\frac{1}{4}\left(F_{\mu \nu}^{(V)}\right)^{2}-\frac{1}{4}\left(\partial_{\mu} \mathcal{B}_{\nu}-\partial_{\nu} \mathcal{B}_{\mu}\right)^{2}+\frac{1}{4} \operatorname{tr}\left(\partial_{\mu} U \partial^{\mu} U^{\dagger}\right)+\frac{1}{2} m_{\rho}^{2} V_{\mu}^{2}-e g_{\rho} V_{3}^{\mu} \mathcal{B}_{\mu}+\frac{1}{2} m_{\mathcal{B}} \mathcal{B}_{\mu}^{2}
$$




$$
+g_{\rho \pi \pi} V^{\mu} \cdot\left(\pi \times \partial_{\mu} \pi\right)+g_{\gamma \pi \pi} \mathcal{B}^{\mu}\left(\pi \times \partial_{\mu} \pi\right)_{3}+\mathcal{L}_{>3}
$$

where the parameters are given as $m_{\rho}^{2}=a g^{2} f_{\pi}^{2}, g_{\rho}=a g f_{\pi}^{2}, g_{\rho \pi \pi}=\frac{1}{2} a g, g_{\gamma \pi \pi}=\left(1-\frac{1}{2} a\right) e$. For $a=2$, these formula are well known to automatically give the universality of the $\rho$ couplings, KSRF relations (KSRF I $: g_{\rho}=2 f_{\pi}^{2} g_{\rho \pi \pi}$, KSRF II; $m_{\rho}^{2}=2 g_{\rho \pi \pi}^{2} f_{\pi}^{2}$ ) and the $\rho$ meson dominance of the electromagnetic form factor of the pion $\left(g_{\gamma \pi \pi}=0\right)$.

First, let us examine the consistency of the hidden local symmetry approach with the low energy theorem. We need to calculate the low temperature correction to $g_{\rho}$. This can be obtained by calculating the one-loop correction and considering the thermal correction coming from pions only [12]. In general there are two types of loops. The first tadpole type of contribution can be obtained by looking at the higher non-derivative pion terms appearing in $\mathcal{L}_{>3}$. Its temperature effect can be easily calculated by forming a pion tadpole loop and inserting the temperature dependent part of the thermal pion propagator $i D_{\pi}^{T}(p)=$ $i D_{\pi}^{T=0}(p)+2 \pi n_{B}\left(p_{0}\right) \delta\left(p^{2}\right)$. This leads to the following simple formula for the correction to a general interaction term involving the $\pi, \rho, \mathcal{B}$

$$
f(\pi, V, \mathcal{B}) \longrightarrow\left[1+\epsilon\left(d / d \pi^{a}\right)^{2}\right] f(\pi, V, \mathcal{B}) .
$$

This clearly breaks chiral symmetry because the pion loop expansion is not consistent with the chiral symmetry, but this breaking is in the next higher order and we need not mind it in the lowest-order calculation. For the non-tadpole type of diagrams, each loop has to be calculated separately.

There is a temperature dependent correction for the photon- $\rho$ meson coupling by the tadpole type of diagram considered in Fig. 1. a. Its contribution can be easily obtained by looking at the photon- $\rho-\pi-\pi$ coupling and using eq. (12);

$$
g_{\rho}(T)=g_{\rho}\left(1-\frac{\epsilon}{2}\right) .
$$

For $\omega$ meson one can explicitly see that such a coupling cancels. The $\rho$ meson mass is modified by the diagram shown in Fig. 2. However, its contribution is of order $\epsilon^{2}$ because of the presence of the derivative in the $\rho \pi \pi$ coupling. We obtain the correct low temperature 
dependence of $g_{\rho}$ and $m_{\rho}$. This is a crucial low temperature theorem that any model with explicit vector meson has to satisfy. In the extended version with explicit $a_{1}$ meson, there exist an additional photon- $a_{1}-\pi$ coupling, so that the $a_{1}$ meson pole also appears as in eq.(5)

Now let us discuss how the low temperature theorem is satisfied in the MYMA. The Lagrangian looks as follows.

$$
\mathcal{L}=\frac{1}{4} f_{\pi}^{2} \operatorname{tr}\left[D_{\mu} U D^{\mu} U^{\dagger}\right]+\frac{1}{2} m_{\rho}^{2} \operatorname{tr}\left[A_{\mu}^{L} A^{\mu L}+A_{\mu}^{R} A^{\mu R}\right]
$$

where,

$$
D_{\mu} U=\partial U-i g A_{\mu}^{L} U+i g U A_{\mu}^{R}
$$

and $A_{\mu}^{L}=V_{\mu}+A_{\mu}, A_{\mu}^{R}=V_{\mu}-A_{\mu}$ and $U=\exp \left[2 i \pi / f_{\pi}\right]$. This with the vector meson dominance, the external vector field $V_{\mu}$ will be identified with the vector meson fields by $V_{\mu}=g_{\rho} \rho_{\mu}$. The relevant terms needed to determine the change in rho meson properties are,

$$
\begin{aligned}
\mathcal{L}= & g^{2} f_{\pi} \epsilon^{a b c} \pi^{a} \rho_{b}^{\mu} a_{c}^{\mu}+\frac{g^{2}}{2}\left[\rho_{\mu}^{2} \pi^{2}-\rho^{\mu} \cdot \pi \rho_{\mu} \cdot \pi\right] \\
& +\frac{1}{2}\left(f_{\pi}^{2} g^{2}+m_{\rho}^{2}\right) a_{\mu}^{2}+\frac{1}{2} m_{\rho}^{2} \rho_{\mu}^{2}+\cdots
\end{aligned}
$$

Here the $a_{1}$ mass is given by $m_{a}^{2}=m_{\rho}^{2}+f_{\pi}^{2} g^{2}$ (Weinberg relation). The main difference with the HGA is that here there is a $\rho \rho \pi \pi$ coupling which at first sight gives a change in the $\rho$ meson mass to order $\epsilon$. However, this contribution has to be added by the $\rho$ meson self energy with an intermediate $a_{1}$ and $\pi$. Together with the rho meson propagator, these contribute to eq.(4) as follows to leading order in $\epsilon$

$$
\begin{aligned}
\Pi_{\mu \nu}^{V}(q, T) & =\left(g_{\mu \nu}-\frac{q_{\mu} q_{\nu}}{m_{\rho}^{2}}\right) g_{\rho}^{2}\left[\frac{i}{m_{\rho}^{2}-q^{2}}+\frac{i}{m_{\rho}^{2}-q^{2}} i g^{2} f_{\pi}^{2} \epsilon \frac{i}{m_{\rho}^{2}-q^{2}}-\frac{i}{m_{\rho}^{2}-q^{2}} \frac{i^{2} g^{4} f_{\pi}^{4} \epsilon}{m_{a}^{2}-q^{2}} \frac{i}{m_{\rho}^{2}-q^{2}}\right] \\
& =\left(g_{\mu \nu}-\frac{q_{\mu} q_{\nu}}{m_{\rho}^{2}}\right) g_{\rho}^{2}\left[(1-\epsilon) \frac{i}{m_{\rho}^{2}-q^{2}}+\epsilon \frac{i}{m_{a}^{2}-q^{2}}\right] .
\end{aligned}
$$

Again vector correlator shows mixing phenomena at finite temperature and satisfies the low temperature theorem. However, it should be noted that in this, if we did not include the $a_{1}$ meson, we would have erroneously obtained a $\rho$ meson mass shift to order $\epsilon$. Whereas in the 
HGA, the low temperature theorem for the $\rho$ meson are satisfied with only the $\rho$ included. This is so because in the HGA, the $\rho$ is a gauge particle of the hidden gauge group and independent of the external left- and right- chiral symmetry, whereas in the MYMA, $\rho$ and $a_{1}$ is related via chiral transformation.

Let us discuss thermal dilepton production from a pion gas [20]. The electromagnetic current is defined as,

$$
J_{\mu}^{e m}=V_{\mu}^{\rho}+\frac{1}{3} V_{\mu}^{\omega}-\frac{\sqrt{2}}{3} V_{\mu}^{\phi} .
$$

The relevant quantity is the thermal average of the retarded correlator of the electromagnetic current, which is related to the time ordered correlator by $\operatorname{Im}\left[\Pi_{\mu \mu}^{F}\right]=\tanh ^{-1}(E / 2 T) \operatorname{Im}\left[\Pi_{\mu \mu}^{R}\right]$. In the soft pion limit, this is a function of only the invariant mass and therefore, the thermal dilepton emission rate per invariant mass can be written as

$$
\frac{d R}{d M^{2}}=\alpha_{e m}^{2} 4 T^{2} \operatorname{Im}\left[\Pi^{F}\left(M^{2}\right)\right] B_{1}(M / T)
$$

where $B(y)=\frac{1}{2 \pi^{2}} \int_{y}^{\infty} d x \sqrt{x^{2}-y^{2}} /\left(e^{y}+1\right)$. Due to mixing, the imaginary part of the time ordered correlator is given by,

$$
\begin{aligned}
\operatorname{Im}\left[\Pi^{V}\left(M^{2}, T\right)\right] & =(1-\epsilon) \frac{f_{\rho}^{2} \Gamma_{\rho} m_{\rho}}{\left(M^{2}-m_{\rho}\right)^{2}+\Gamma_{\rho}^{2} m_{\rho}^{2}}+\text { continuum } \\
\operatorname{Im}\left[\Pi^{S V}\left(M^{2}, T\right)\right] & =\frac{f_{\omega}^{2} \Gamma_{\omega} m_{\omega}}{\left(M^{2}-m_{\omega}\right)^{2}+\Gamma_{\omega}^{2} m_{\omega}^{2}}+\text { continuum }
\end{aligned}
$$

in the soft pion limit near the resonance region. Here, for simplicity, we have used a BreitWigner parameterization and $m_{\rho}=770 \mathrm{MeV}, \Gamma_{\rho}=150 \mathrm{MeV}, f_{\rho}=152 \mathrm{MeV}$ and $m_{\omega}=782$ $\mathrm{MeV}, \Gamma_{\omega}=9.8 \mathrm{MeV}, f_{\omega}=46 \mathrm{MeV}$.

In principle, the ratio of peak height between the $\rho$ meson and $\omega$ meson should decrease as $1-\epsilon$. In Fig. 3, we have plotted the expected thermal dilepton production eq.(19) at $T=100$ and $150 \mathrm{MeV}$, above which the leading order expression should break down. The origin of the relative reduction of the $\rho$ peak in the dilepton spectrum is coming from the medium dependent decay constant, which is related to its $e^{+} e^{-}$partial width as $g_{\rho}^{2}=$ $3 m_{\rho} \Gamma\left(\rho \rightarrow e^{+} e^{-}\right) / 4 \pi \alpha^{2}$. Because of this reason, even $\rho$ meson produced in the initial stage 
of the collision will also be affected as long as its decay into dileptons takes place inside the thermal pion gas.

In previous experiment, it was not possible to separate $\rho$ and $\omega$ peaks in the dilepton spectrum of RHIC [21]. Also, it is not clear how the leading temperature effects should modify at higher temperature. However, present hadronic model calculations to understand the NA 38 data at CERN [22] on $J / \Psi$ suppression and $\phi /(\rho+\omega)$ enhancement in $\mathrm{O}+\mathrm{U}$ and $\mathrm{S}+\mathrm{U}$ collision is consistent with an overall reduction of $\rho+\omega$ observed in the dilepton spectrum. Koch, Heinz and Pišút [23] first obtained a relation between the transverse energy $\left(E_{T}\right)$ and the fireball size or impact parameter by fitting it to the $J / \Psi$ suppression with increasing $E_{T}$. The suppression comes mainly from $J / \Psi$ absorption. Then assuming that the ratio of $\phi / \omega$ production from rescattering is the same as in the primary collisions, using the fact that $\phi$ absorption cross section is less than that of the $\omega$ and the same relation between $E_{T}$ and impact parameter, they were able to explain the $\phi$ enhancement data. However, taking into account secondary $\rho$ production, the fit deteriorates unless there is an additional mechanism to reduce the rho meson decaying into dileptons [23,24]. In fact, the original data are even consistent with a systematic reduction of $N_{\rho}+N_{\omega}$ in A A collision compared to $N_{\rho}+N_{\omega}$ in p A collision times A [22].

In summary, we have shown that both effective chiral model of hidden gauge approach and massive Yang-Mills approach are consistent with the mixing properties of the vector current correlator at low temperature. We have also discussed some phenomenological consequence expected in the dilepton channel in the $\rho$ and $\omega$ resonance region.

We would like to thank M. Lutz for emphasizing the equivalence between the HGA and MYMA. We would like to thank T. Hatsuda, C.M. Ko, G. A. Miller M. Rho for useful comments. The work of SHL was supported in part by the Basic Science Research Institute Program, Ministry of Education of Korea, no. BSRI-94-2425 and The US Department of Energy. The work of CS was supported by the National Science Foundation under Grant No. PHY-9212209 and Welch Foundation under Grant No. A-1110. SHL and HY would like to thank B. Fridman and W. Noerenberg for inviting to the ECT* workshop on "Mesons and 
Baryons in Hadronic Matter" where part of this work was done and for useful discussions. 


\section{REFERENCES}

* e-mail: suhoung@phya.yonsei.ac.kr

† e-mail: song@comp.tamu.edu

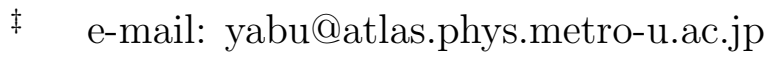

[1] Quark Matter '93, Nucl. Phys. A 556 (1994) 1.

[2] R. Pisarski, Phys. Lett. B 160 (1982) 222.

[3] M. Dey, V. L. Eletsky and B. L. Ioffe, Phys. Lett. B 252 (1990) 620.

[4] C. Gale and J. I. Kapusta, Nucl. Phys. B 357 (1991) 65;

C. Song, Phys. Rev. D 48 (1993) 1375;

C. Song, C. M. Ko and C. Gale, Phys. Rev. D 50 (1994) R1.

[5] A. I. Bochkarev and M. E. Shaposhnikov, Nucl. Phys. B 268 (1986) 220;

H. G. Dosch and S. Narison, Phys. Lett. B 203 (1988) 155.

[6] R. Furnstahl, T. Hatsuda and Su H. Lee, Phys. Rev. D 42 (1990) 1744;

C. A. Dominguez and M. Loewe, Phys. Lett. B 233 (1989) 201.

[7] T. Hatsuda, Y. Koike and Su H. Lee, Nucl. Phys. B 394 (1993) 221; Phys. Rev. D 47 (1993) 1225.

[8] T. Hatsuda and Su H. Lee, Phys. Rev. C 46 (1992) R34.

[9] V. L. Eletsky and B. L. Ioffe, Phys. Rev. D 47 (1993) 3083.

[10] S. Weinberg, Physica A 96 (1979) 327.

[11] J. Gasser and H. Leutwyler, Ann. Phys. (NY) 158 (1984) 142; Nucl. Phys. B 250 (1985) 465.

[12] J. Gasser and H. Leutwyler, Phys. Lett. B 184 (1987) 83;

P. Gerber and H. Leutwyler, Nucl. Phys. B 321 (1989) 387. 
[13] H. Leutwyler and A. V. Smilga, Nucl. Phys. B 342 (1990) 302;

V. L. Eletsky, Phys. Lett. B 245 (1990) 229.

[14] J. J. Sakurai, Currents and Mesons (Chicago, Chicago, 1969).

[15] M. Bando, T. Kugo and K. Yamawaki, Phys. Rep. 164 (1988) 217.

[16] K. Yamawaki, Phys. Rev. D 35 (1987) 412.

[17] U.-G. Meißner and I. Zahed, Z. Phys. A 327 (1987) 5;

U.-G. Meißner, Phys. Rep. 161 (1988) 213.

[18] K. Yamawaki, Proc. of 1987 International Workshop on Low Energy Effective Theory of QCD, Nagoya 1987. editor S. Saito and K. Yamawaki.

[19] S. Coleman, J. Wess and B. Zumino, Phys. Rev. D 177 (1969) 2239;

C. G. Callan, J. Coleman, J. Wess and B. Zumino, Phys. Rev. D 177 (1969) 2247.

[20] For a review on dilepton spectrum from QGP see, P. V. Ruuskanen in Quark-Gluon Plasma edited by R. Hwa. (World Scientific, Singapore, 1990).

[21] In future RHIC at Brookhaven, this resolution is possible. We thank T. Hatsuda for this information.

[22] NA38 collaboration, C. Baglin et.al., Phys. Lett. B 272 (1991) 449.

[23] P. Koch, U. Heinz and J. Pišút, Phys. Lett. B 243 (1990) 149; Z. Phys. C 47 (1990) 477.

[24] U. Heinz and K. S. Lee, Phys. Lett. B 259 (1991) 162. 


\section{Figure Captions}

Fig. 1 One-loop corrections to correlators in the thermal pion gas. Solid line denotes the zero temperature vector correlator and double solid line does the axial vector correlator. Dashed lines correspond to thermal pions.

Fig. 2 One loop diagram for the $\rho$ meson self-energy.

Fig. 3 Thermal dilepton production rate in $\mathrm{fm}^{-4} \mathrm{GeV}^{-2}$. The solid line is the result obtained with low temperature correction. 
This figure "fig1-1.png" is available in "png" format from: http://arxiv.org/ps/hep-ph/9408266v1 
This figure "fig1-2.png" is available in "png" format from: http://arxiv.org/ps/hep-ph/9408266v1 
This figure "fig1-3.png" is available in "png" format from: http://arxiv.org/ps/hep-ph/9408266v1 